\title{
El gran colisionador de hadrones: una maravilla del mundo moderno
}

\author{
Lizardo Valencia Palomo
}

\section{Resumen}

Para estudiar los constituyentes básicos de la materia, es necesario construir aceleradores de partículas que sean capaces de recrear, a escalas atómicas, los primeros instantes del inicio del universo. El Gran colisionador de hadrones es la máquina más grande que el ser humano haya construido. Una máquina cuyas características tecnológicas la convierten en una maravilla del mundo moderno.

El presente artículo tiene como objetivo que el lector conozca algunos de los detalles más asombrosos del Gran colisionador de hadrones. De igual forma, se aprenderán conceptos relacionados con la física de partículas elementales.

Palabras clave: CERN, LHC, física de partículas, tecnología.

\section{The Large Hadron Collider: a marvel of the modern world}

\begin{abstract}
In order to study the fundamental blocks of nature, it is necessary to build particle accelerators. These machines are able to recreate, at an atomic level, the first instants after the birth of our universe. The Large Hadron Collider is the largest machine ever built by humanity. Its technological characteristics make it a marvel of the modern world.

The goal of the present paper is to show some of the most astonishing details about the Large Hadron Collider. In this way, the reader will also learn basic concepts related to the field of elementary particle physics.
\end{abstract}

Keywords: CERN, LHC, particle physics, technology.

Recepción: 30/11/2020. Aprobación: 30/04/2021. Dol: http://doi.org/10.22201/cuaieed.16076079e.2021.22.5.4 
"El gran colisionador de hadrones: una maravilla del mundo moderno" Lizardo Valencia Palomo

Vol. 22, Núm. 5, septiembre-octubre 2021 Revista Digital Universitaria

\section{Lizardo Valencia Palomo}

lizardo.valencia@unison.mx

Realizó sus estudios de Licenciatura en Física en la Facultad de Ciencias de la Universidad Nacional Autónoma de México (UnAm) y su Maestría en Ciencias (Física) en el Instituto de Física de esa casa de estudios. En el 2010 fue contratado por el Instituto de Física Nuclear de la Universidad de París Sur en Francia para realizar sus estudios de Doctorado. Tres años después fue contratado por el Consejo Nacional de la Investigación Científica de Francia para realizar un posdoctorado en la Universidad Blaise Pascal en Clermont-Ferrand. En 2015 regresó a México gracias al Programa de Repatriaciones del conacrt, al año siguiente obtuvo el nombramiento de Profesor de Tiempo Completo en la Facultad de Física y Matemáticas de la Universidad Autónoma de Chiapas. Desde el 2017 se desempeña como Profesor-Investigador en el Departamento de Investigación en Física de la Universidad de Sonora. Ha trabajado los últimos 13 años en experimentos del Gran colisionador de hadrones (LHC) del CERN. Actualmente es miembro del Sistema Nacional de Investigadores nivel 1.

\section{Introducción}

La física de partículas estudia los bloques fundamentales de la materia. Al entender a la naturaleza en sus dimensiones más pequeñas es posible llegar a comprender al universo en sus dimensiones más grandes, ya que, entre otras cosas, se puede explicar su origen y expansión. Para el estudio de las partículas fundamentales de la materia se requiere de instrumentos especializados: Ios aceleradores de partículas, con los cuales se logra acelerar partículas a energías muy altas, de ahí que a este campo de estudio también se le conozca como física de altas energías.

Para poder cuantificar a la naturaleza en toda su escala, se necesita del lenguaje de las matemáticas. Por esta razón, al momento de describir las propiedades (masa, tamaño, etcétera) de diversos objetos, es más práctico el uso de la notación científica. Esta forma de expresar números se basa en el uso de las potencias de diez (ver tabla 1).

Tabla 1. La columna de la izquierda contiene números (enteros y decimales), la columna central muestra cómo se escribe el número en notación científica y la columna de la derecha indica el prefijo y símbolo correspondiente (CERN Education, Communications and Outreach Group, 2017, p. 1).

\begin{tabular}{|c|c|c|}
\hline Número & Notación científica & Prefijo (símbolo) \\
\hline 0.000000000000000001 & $10^{-18}$ & Atto $(\mathrm{a})$ \\
\hline 0.000000000000001 & $10^{-15}$ & Femto $(\mathrm{f})$ \\
\hline 0.0000000001 & $10^{-10}$ & Angstrom $(\AA)$ \\
\hline 0.000001 & $10^{-6}$ & Micro $(\mu)$ \\
\hline 0.001 & $10^{-3}$ & Mili $(\mathrm{m})$ \\
\hline 1 & $10^{0}$ & Kilo $(\mathrm{k})$ \\
\hline 1,000 & $10^{3}$ & Mega $(\mathrm{M})$ \\
\hline $1,000,000$ & $10^{6}$ & Giga $(\mathrm{G})$ \\
\hline $1,000,000,000$ & $10^{9}$ & Tera $(\mathrm{T})$ \\
\hline $1,000,000,000,000$ & $10^{12}$ & Peta $(\mathrm{P})$ \\
\hline $1,000,000,000,000,000$ & $10^{15}$ & \\
\hline
\end{tabular}


Figura 1. Interior del Gran colisionador de hadrones. CERN copyright.

\footnotetext{
1.Partículas que componen a los
} protones y neutrones.
Para tener una idea de las dimensiones de los objetos más pequeños que encontramos en la naturaleza, podemos decir que las células miden aproximadamente un micrómetro $\left(10^{-6} \mathrm{~m}\right)$, el átomo es del tamaño de un angstrómetro $\left(10^{-10} \mathrm{~m}\right)$, su núcleo mide unos cuantos femtómetros $\left(10^{-15} \mathrm{~m}\right)$ y los quarks ${ }^{1}$ son incluso más pequeños que un attómetro (ver tabla 1).

\section{El gran colisionador de hadrones}

El gran colisionador de hadrones (LHC) es un acelerador de partículas que mide 27 kilómetros de circunferencia y que se localiza en la frontera entre Suiza y Francia (ver figura 1). Esta enorme máquina se encuentra a 100 metros bajo tierra y fue construida por la Organización Europea para la Investigación Nuclear (CERN).

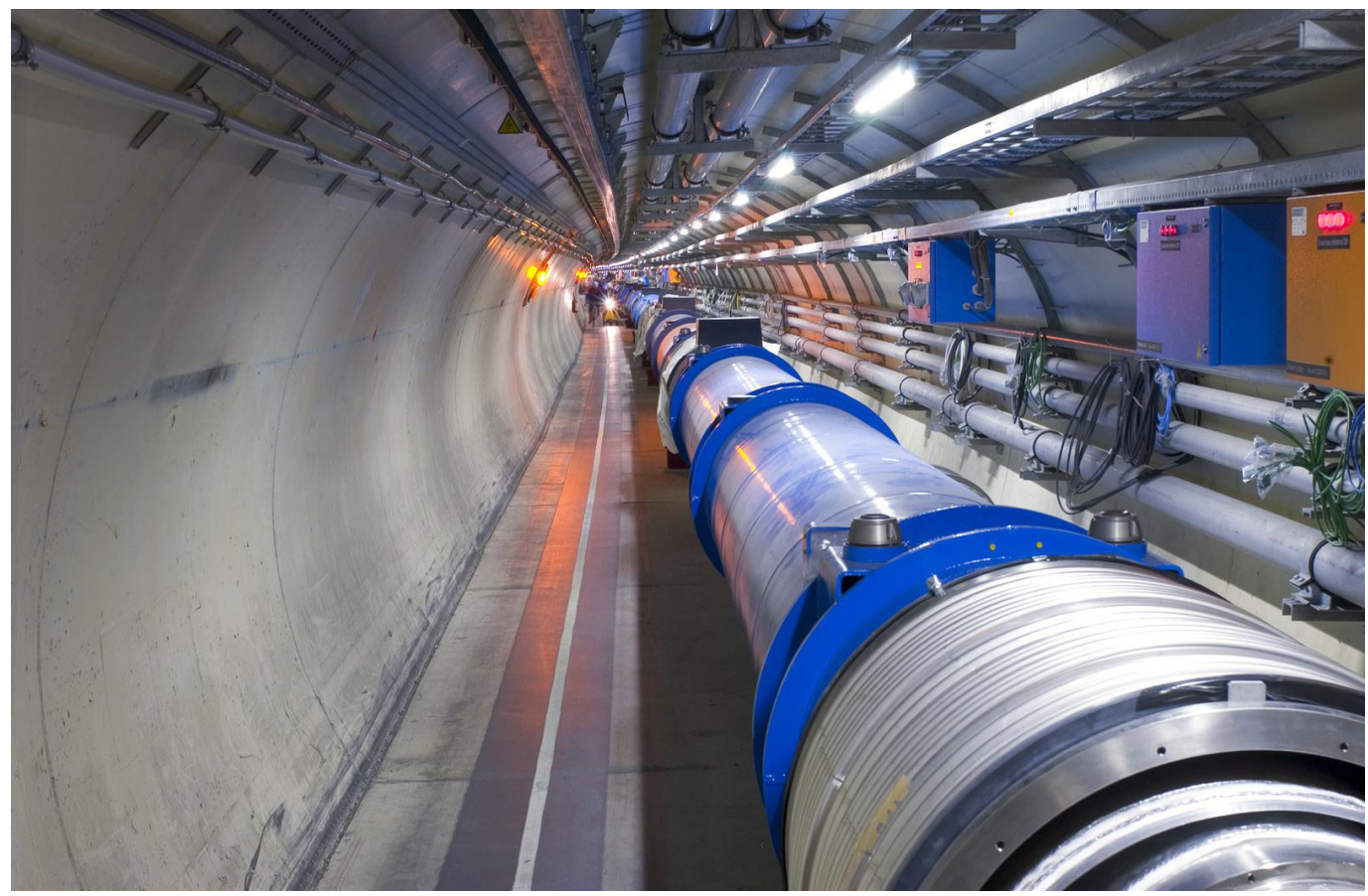

Es 'gran' debido a su formidable tamaño, de hecho, es el acelerador de partículas más grande del mundo. Es 'colisionador' porque no sólo acelera las partículas, también las hace colisionar unas contra otras. Los hadrones son todas aquellas partículas compuestas por quarks, como por ejemplo los protones y neutrones que conforman el núcleo atómico. De hecho, lo que el LHc colisiona son protones y núcleos atómicos.

Oficialmente se considera que los planes para el desarrollo del LHc iniciaron con un simposio que se llevó a cabo en Lausana (Suiza) en 1984. Ahora bien, las primeras colisiones entre protones en el LHC se dieron en noviembre de 2009. Por lo tanto, estamos hablando que tuvieron que transcurrir 25 años de planeación y desarrollo para que esta maravilla del mundo moderno empezara a funcionar. 
Debido a sus altos costos, en un principio se había pensado que el LHC se construiría en dos etapas. Sin embargo, gracias a que Japón, Estados Unidos, India y otros países que también contribuyeron con los gastos, el proyecto se realizó en una sola fase.

El costo material total del LHC fue de más de 4,000 millones de francos suizos, lo que se traduce en casi 100 mil millones de pesos mexicanos (Evans, 2018, p. 42). Sin embargo, esto no toma en cuenta los gastos en la construcción del túnel en el que se encuentra, ya que esta obra de ingeniería ya existía.

\section{La punta del iceberg}

Pero el LHC es tan sólo el último eslabón de toda una cadena de aceleradores previos, sin los cuales éste no podría funcionar (ver figura 2). Antes de que el LHC empezara sus operaciones, el cERn, a lo largo de más de 50 años, construyó muchos otros aceleradores de partículas. Cada uno de ellos se construyó de un tamaño mayor al de su predecesor, de tal forma que la velocidad a la que se aceleran las partículas aumentara.

Figura 2. Complejo de aceleradores del CERN. La trayectoria de los protones hacia el LHc es señalada con flechas color gris claro. cERN copyright.

\section{CERN's Accelerator Complex}

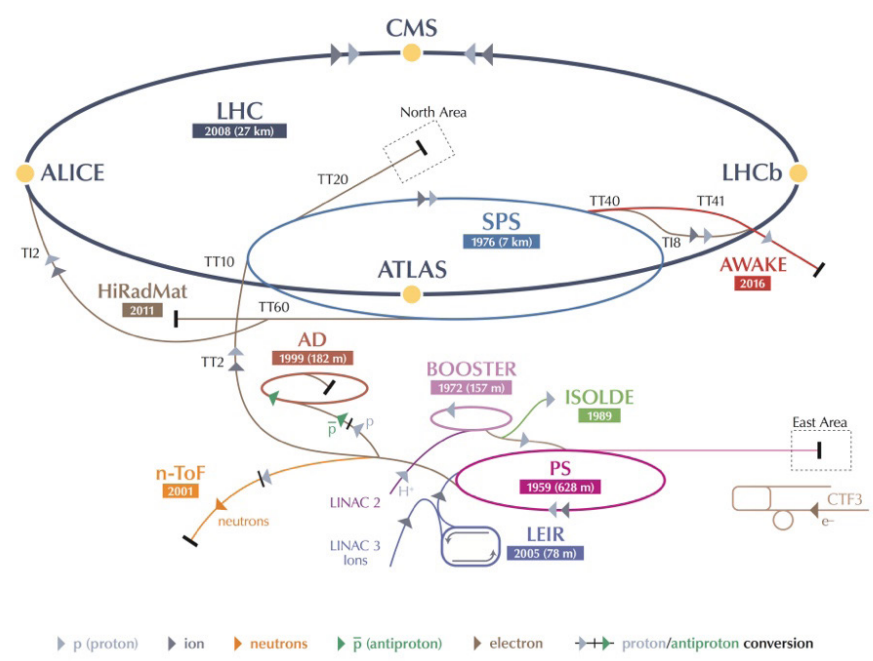

LHC Large Hadron Collider SPS Super Proton Synchrotron PS Proton Synchrotron

AD Antiproton Decelerator CTF3 Clic Test Facility AWAKE Advanced WAKefield Experiment ISOLDE Isotope Separator OnLine DEvice LEIR Low Energy lon Ring LINAC LINear ACcelerator n-ToF Neutrons Time Of Flight HiRadMat High-Radiation to Materials

Existen dos tipos de aceleradores que preceden al LHC, que por su forma geométrica son catalogados como aceleradores lineales y circulares. Para las colisiones entre protones el camino empieza en una botella que contiene hidrógeno. A cada uno de estos átomos se les arranca el único electrón que orbita el núcleo, formado únicamente por un protón. De esta forma, los protones 
primero recorren los $80 \mathrm{~m}$ de un acelerador lineal y luego son inyectados a un primer acelerador circular de 160 m de circunferencia. Aquí las partículas se mantienen girando a través de cuatro anillos hasta que se reúne una importante cantidad de protones. Posteriormente éstos son enviados a otro acelerador circular de $630 \mathrm{~m}$ de circunferencia, en donde los protones son agrupados en paquetes. Por último, los protones recorren un tercer acelerador circular que mide $7 \mathrm{Km}$ de circunferencia y de ahí son transferidos al LHC.

\section{Energía y velocidad de la luz}

En la física de partículas las energías utilizadas son muy pequeñas comparadas con las energías que encontramos en nuestra vida diaria. Por esta razón se emplea el electrón-volt (eV) como unidad de medida, que se define como la energía que adquiere un electrón al ser acelerado por una diferencia de potencial de un volt. Además, en un acelerador las partículas se desplazan a velocidades muy altas, tanto así que para tener una mejor visión comparamos dichas velocidades con la velocidad de la luz en el vacío (299,792 kilómetros/segundo). Esto es así porque en el vacío ningún objeto material puede alcanzar la velocidad a la que se desplaza la luz (denotada por c). Es importante entender que se requiere de una cantidad de energía muy grande, en electrón-volts, para que una partícula alcance una velocidad cercana a la de la luz.

Conociendo la energía y masa de una partícula, es posible calcular la velocidad a la que se desplaza. De esta forma, con la energía a la que son acelerados los protones en cada acelerador de partículas, podemos calcular su velocidad (ver tabla 2).

Tabla 2. Relación existente entre la energía y velocidad (en porcentaje de la velocidad de la luz) de un protón al recorrer los diferentes aceleradores que lo conducen hasta el LHC (CERN Education, Communications and Outreach Group, 2017, p. 5).

\begin{tabular}{|c|c|c|}
\hline Acelerador & Energía & Velocidad (\% de $c$ ) \\
\hline LINAC 2 & $50 \mathrm{MeV}$ & 31.4 \\
\hline PSB & $1.4 \mathrm{GeV}$ & 91.6 \\
\hline PS & $25 \mathrm{GeV}$ & 99.93 \\
\hline SPS & $450 \mathrm{GeV}$ & 99.9998 \\
\hline LHC & $7 \mathrm{TeV}$ & 99.9999991 \\
\hline
\end{tabular}

\section{El congelador más grande del mundo}

La razón de tener aceleradores circulares cada vez más grandes radica en el hecho de que conforme las partículas se aceleran adquieren mayor energía. Esto se traduce en un incremento de la órbita en la que se mueven. Es aquí en donde entran en juego los imanes, ya que éstos generan campos magnéticos que mantienen a las partículas en una órbita fija. Por consiguiente, si se quisiera aumentar la energía a la que el ıHc acelera las partículas hay dos opciones: hacer más grande su circunferencia o que sus imanes generen un campo magnético más intenso.

En el ıHc hay más de 9,500 imanes y entre ellos hay de diferentes tipos, que los podemos dividir en dos grupos. Por un lado, están los dipolos que mantienen a las partículas en su órbita circular y por otro lado están aquellos que sirven 
para compactar el haz de partículas. Cuando el LHc acelera los protones a su máxima energía, los dipolos deben generar un campo magnético 100,000 veces más intenso que el campo magnético terrestre para mantener a las partículas en una órbita estable (cERN engineering, 2021).

Producir este campo magnético tan intenso requiere que los dipolos estén hechos de cables superconductores, es decir, material que conduce corriente eléctrica sin resistencia. Pero para lograr esto, dichos cables deben funcionar a temperaturas muy bajas, por lo que en el LHC son enfriados a $-271^{\circ} \mathrm{C}$, convirtiendo a este acelerador de partículas en el congelador más grande del planeta. Una vez estabilizados a dicha temperatura, se hace circular una corriente de más de 11,000 Amperios en los imanes, que produce a su vez el campo magnético. Tomando en cuenta que una corriente eléctrica de apenas 1 Amperio puede llegar a ser fatal en el ser humano, vemos que la cantidad de corriente que se hace circular en el LHC es enorme y que además debe de ser tratada con mucho cuidado.

Ahora bien, para lograr estas temperaturas tan bajas se utiliza helio líquido. La tabla periódica de los elementos indica que en la naturaleza el helio se encuentra como gas, pero al enfriarlo a poco menos de $-270{ }^{\circ} \mathrm{C}$ se vuelve líquido. Si se continúa enfriando, el helio líquido se convierte en un superfluido, por lo que fluye sin fricción. En este estado, el helio líquido se convierte en el congelante óptimo para refrigerar y estabilizar grandes sistemas de superconductores.

\section{Un tren de alta velocidad}

Imagina que estás en la playa, tocas la arena y observas los finos granos que la conforman. Al verlos tan pequeños piensas en lo difícil que sería lograr que dos granitos de arena choquen al ser lanzados uno contra otro. Se te ocurre entonces que, para aumentar la probabilidad de que dos de estos granos choquen, sería mejor agarrar dos puñados de arena y aventarlos entre sí. Pero al hacer esto notas que cuando arrojas la arena, ésta se expande en el aire justo al abandonar tu mano. Comprendes así que lo mejor sería que ambos puñados de arena se encuentraran lo más compactos posibles justo antes de chocar. De esta forma aumentaría, aún más, la probabilidad de que, al menos, dos granitos de arena choquen.

Un razonamiento similar se aplica en los aceleradores de partículas. En el caso del LHC se pueden agrupar $10^{11}$ protones para formar un paquete, de tal forma que cada haz que circula por esta máquina puede llegar a estar compuesto de más de 2,800 paquetes. Justo antes de hacer chocar estos haces, los paquetes son compactados hasta que llegan a tener una anchura menor a la mitad del grosor de un cabello humano (50 micrómetros).

Con todos estos paquetes y protones que los conforman, cada haz que circula en el LHC contiene la misma energía que un tren de 400 toneladas viajando a 150 kilómetros/hora (LHC machine, 2021). Cuando el haz se encuentra girando en el acelerador, debe ser minuciosamente monitoreado para prevenir terribles accidentes. 
Al mismo tiempo, es importante que el haz, con toda esta energía, sólo colisione con el otro haz en los puntos especialmente diseñados y adaptados para ello. Por consiguiente, es necesario que la tubería por la que circula esté libre de moléculas de gas residual. Hablamos, entonces, de que dichos conductos están a un alto vacío.

\section{La energía de un mosquito}

Funcionando a su máxima capacidad, el LHc hará colisionar protones a energías nunca antes alcanzadas en ningún otro acelerador de partículas. Estas colisiones serán tan violentas que podrán recrear los primeros instantes del inicio del universo. Para tener una mejor idea de lo energéticas que serán estas colisiones, podemos decir que justo antes de colisionar, los protones tendrán una energía equivalente a la que tiene un mosquito en pleno vuelo (Symmetry Magazine, 2021).

Sí, leíste bien. El gran colisionador de hadrones, cuyo costo fue de casi 100 mil millones de pesos mexicanos realiza colisiones a las energías equivalentes al choque entre dos mosquitos. Si esto es verdad, ¿por qué entonces decimos que el LHc es una maravilla tecnológica? Pues bien, lo que hace al LHc tan especial es la concentración de energía. Si bien es cierto que la energía de los protones es la misma que emplea un mosquito para volar, las partículas subatómicas son billones de veces más pequeñas. Esto es lo realmente extraordinario del LHC.

Figura 3. Una colisión protónprotón en un experimento del LHC. CERN cOpyright.

Cuando dos protones colisionan, la energía da lugar a la creación de nuevas partículas que salen disparadas del punto de colisión. Mientras más energética

CMS Experiment at the LHC, CERN

Data recorded: 2016-Jun-05 03:23:15.108257 GMT

Run / Event / LS: 274422 / 979073892 / 558 sea la colisión, se producirán más partículas y con mayor velocidad. En estos momentos uno bien podría preguntarse: ¿cuántas colisiones se generan cada segundo en el LHC? Saquemos cuentas. Existen $10^{11}$ protones en cada uno de los poco más de 2,800 paquetes que conforman cada haz, los paquetes se cruzan 30 millones de veces cada segundo y cada vez que esto sucede se generan en promedio 40 colisiones. Por consiguiente, estamos hablando que en el LHC se generan aproximadamente mil millones de colisiones cada segundo (ver figura 3). 


\section{Datos, datos y más datos}

Las partículas que se producen en todas estas colisiones atraviesan los detectores de los experimentos. Los circuitos electrónicos registran el paso de dichas partículas como señales electrónicas y envían la información al Centro de Datos del cenn para la reconstrucción digital. No obstante, es imposible procesar y guardar la información correspondiente a todas las colisiones que se producen en el LHc. Por consiguiente, se emplea un filtro de toda esta información, de tal forma que la cantidad de datos se reduce drásticamente y sólo se seleccionan aquellos eventos que sean interesantes para su estudio posterior: tan sólo 1\% de todas las colisiones.

Aún después de esta selección, los experimentos del lHc generan unos 90 petabytes de datos al año, de los cuales el Centro de Datos del cern procesa y almacena un tercio de todo eso. Para darnos una idea, tan sólo con lo almacenado podríamos Ilenar más de 1.2 millones de discos Blu-ray, equivalentes a reproducir 250 años de video en alta definición sin interrupciones (CERN facts, 2021).

Para solventar el problema de almacenamiento y procesamiento de datos, se creó la Red Mundial de Cómputo del LHc, una red global de centros de supercómputo interconectados. Esta red está compuesta de unos 900,000 núcleos de procesamiento, distribuidos en 170 centros de supercómputo en 42 países. De esta forma, los 12,000 físicos que trabajan en el LHc pueden analizar los datos generados en cualquier momento (CERN computing, 2021).

\section{Conclusión}

El gran colisionador de hadrones es el acelerador de partículas más grande y más poderoso del mundo. Para lograr esto, se ha utilizado tecnología de punta en todo su diseño: desde los cables superconductores que constituyen los imanes, hasta el enorme poder de supercómputo que se necesita para procesar la gigantesca cantidad de datos que se extraen. Pero como toda máquina, el LHC necesita recibir ajustes y mantenimiento. Es por eso que desde finales de 2018 dejó de funcionar y volverá con renovados bríos a mediados de 2021.

\section{Referencias}

* cern computing. (2021, 15 de mayo). Computing. https://home.cern/science/ computing

- CERN engineering. (2021, 15 de mayo). Engineering. https://home.cern/science/ engineering

- cern facts. (2021, 15 de mayo). Facts and figures about the LHc. https://home.cern/ resources/faqs/facts-and-figures-about-lhc 
* cern Education, Communications and Outreach Group. (2017). Lhc guide. cern.

* Evans, L. (2018). The Large Hadron Collider: a marvel of technology (2. a ed). EPFL Press.

* LHC machine. (2021, 15 de mayo). LHC machine outreach. https://lhc-machineoutreach.web.cern.ch/

* Symmetry Magazine. (2021, 15 de mayo). Inside the Large Hadron Collider. https:// www.symmetrymagazine.org/article/inside-the-large-hadron-collider

\section{Cómo CITAR ESTE ARTículo}

* Valencia Palomo, Lizardo. (2021, septiembre-octubre). El gran colisionador de hadrones: una maravilla del mundo moderno. Revista Digital Universitaria (RDU), 22(5). http://doi.org/10.22201/cuaieed.16076079e.2021.22.5.4 